Review

\title{
Biomass Converting Enzymes as Industrial Biocatalysts for Fuels and Chemicals: Recent Developments
}

\author{
Matt D. Sweeney and Feng Xu \\ Novozymes Inc., 1445 Drew Avenue, Davis, CA 95618, USA; \\ E-Mails: mswn@novozymes.com (M.D.S.); fxu@ novozymes.com (F.X.); \\ Tel.: +1-530-757-8100; Fax: +1-530-758-0317
}

Received: 16 January 2012; in revised form: 18 February 2012 / Accepted: 28 March 2012 /

Published: 12 April 2012

\begin{abstract}
The economic utilization of abundant lignocellulosic biomass as a feedstock for the production of fuel and chemicals would represent a profound shift in industrial carbon utilization, allowing sustainable resources to substitute for, and compete with, petroleum based products. In order to exploit biomass as a source material for production of renewable compounds, it must first be broken down into constituent compounds, such as sugars, that can be more easily converted in chemical and biological processes. Lignocellulose is, unfortunately, a heterogeneous and recalcitrant material which is highly resistant to depolymerization. Many microorganisms have evolved repertoires of enzyme activities which act in tandem to decompose the various components of lignocellulosic biomass. In this review, we discuss recent advances in the understanding of these enzymes, with particular regard to those activities deemed likely to be applicable in commercialized biomass utilization processes.
\end{abstract}

Keywords: biomass conversion; lignocelluloses; cellulose; hemicellulase; oxidoreductase

\section{Introduction}

Converting renewable, widely available yet vastly underused lignocellulosic biomass to valuable chemicals, including fuel and polymer precursors, is of strategic importance for the sustainability and advancement of energy and chemicals industries (for recent reviews, see [1-11]). Compared to the commercialized starch and sugarcane-based "first generation" biofuel (starch or sucrose conversion to ethanol), emergent biomass-based "second generation" biofuel ((hemi)cellulose conversion to ethanol) 
has the potential of not only significantly displacing fossil fuels, but also adding value to agricultural byproducts, forestry residues, or municipal wastes. Biomass conversion is also being expanded beyond fuel production; the concept of biorefinery is being actively pursued so that a wide range of useful materials (for chemicals, energy, food, healthcare, and other industries) can be derived from biomass.

Naturally occurring lignocellulosic biomass, especially in plant cell walls, serves structural and protective roles for plants, and consequently is recalcitrant and resistant to degradation. It is a major challenge to convert or degrade at industrial scale highly complex and heterogeneous lignocellulosic biomass into simple carbohydrates, phenolics, aromatics, and other more transformable substances. Among the numerous physical, chemical, and biological methods under development, the ones relying on enzymes are particularly attractive. Natural lignocellulose degradation and utilization (as part of natural energy transfer and carbon cycle) are carried out by specific enzymes from lignocellulolytic organisms (especially wood-degrading fungi and bacteria). As potential industrial catalysts for biomass conversion, enzymes might provide high specificity, low energy or chemical consumption, or low environment pollution.

Lignocellulosic biomass consists of morphologically different cellulose, structurally and compositionally complex hemicellulose, recalcitrant lignin, diverse proteins, different lipids, and other substances that interact with each other. The primary role of biomass-converting enzymes is to degrade polymeric cellulose or hemicellulose into simple saccharides, sugars which can then be fermented by microorganisms to, or serve as platform molecules for synthesis of valuable fuel or chemicals. Cellulases and hemicellulases can degrade cellulose and hemicellulose to constituent hexoses and pentoses. In general, biomass-converting enzymes have to work in concert, to benefit from synergism among their specificity (towards different components and regions of lignocellulose) as well as mitigation of their inhibition (by different lignocellulose components or degradation products).

Biomass-utilizing organisms, widely distributed in archaea, bacteria, fungi, protists, plants, and animals (including symbiotic gastrointestinal microbes), possess numerous lignocellulolytic enzymes acting on the (hemi)cellulose backbone, hemicellulose substituents, or cellulose-shielding lignin. Many of these enzymes are secreted, either alone or forming supramolecular cellulosome, thus making them promising industrial biocatalysts for biomass conversion. In-depth and systematic basic studies on biomass-active enzymes have been made for decades, and comprehensive reviews for the field have been written (for recent reviews, see [12-22]). In this review, only the most recent developments, especially those relevant to the enzymological aspects of the commercial enzymatic biomass conversion biotechnology, are introduced.

\section{Overview}

The myriad of biomass-active, lignocellulose-degrading enzymes may be classified in ways emphasizing catalyzed reaction (specificity), structural/evolutionary relation, or other aspects. Based on the International Union of Biochemistry and Molecular Biology's Enzyme Nomenclature and Classification (http://www.chem.qmul.ac.uk/iubmb/enzyme/ [23]), these enzymes belong to EC 3.2.1 glycosidases, EC 4.2.2 lyases, EC 3.1.1 esterases, EC 1.11.1 peroxidases, EC 1.1.3 carbohydrate oxidases, EC 1.10.3 phenol oxidase, and other EC classes, according to their main reactions. Each 
class and subclass has shared primary enzyme substrates, a feature that may facilitate enzyme selections for targeted biomass materials.

Based on Carbohydrate-Active EnZYmes (http://www.cazy.org/ [24]) and Fungal Oxidative Lignin Enzymes (FOLy) (http://foly.esil.univ-mrs.fr/ [25]) databases, lignocellulose-degrading enzymes belong to Glycoside Hydrolases (GH), Polysaccharide Lyases (PL), Carbohydrate Esterases (CE), Lignin Oxidases (LO), and Lignin Degrading Auxiliary enzymes (LDA) families according to their sequence and structural homology. Each family has shared three-dimensional structure and catalytic mechanism, a feature that may facilitate bioinformatic analysis of (meta)genomic data. Yet enzymes from different families may catalyze the same reaction.

A distinct structural feature of lignocellulose-degrading enzymes is their modularity. In addition to the catalytic core, many of these enzymes also possess non-catalytic but functionally important domains, including carbohydrate-binding modules (CBM), fibronectin 3-like modules, dockerins, immunoglobulin-like domains, or functionally unknown " $X$ " domains. Having affinity to bundled or individual polysaccharide chains or to single carbohydrate molecules, CBM anchors or directs host enzymes to targeted carbohydrate substrates [26], and in some cases even disrupts crystalline cellulose microfibrils to assist cellulase reaction enzymes [13,27]. Through specific affinity to cohesion, dockerin anchors host enzymes onto scaffoldin to assemble a cellulosome comprising a clustering of different but synergistic/interdependent enzymes [28-30]. Modularity equips lignocellulose-degrading enzymes with vast versatility.

Many lignocellulose-degrading enzymes employ hydrolytic reactions (mainly acting on (hemi)cellulose), while others employ oxidoreductive ones (mainly acting on lignin), to convert lignocellulose. Almost all cellulases and hemicellulases are carbohydrate hydrolases relying on either a "retaining" mechanism, which yields product of the same anomeric configuration after breaking a glycosidic bond with a "double-displacement" hydrolysis, or an "inverting" mechanism, which yields product of the opposite anomeric configuration after breaking a glycosidic bond with a "single nucleophilic-displacement" hydrolysis, both involving two acidic amino acid residues (Glu or Asp) as a proton donor or general acid and as a nucleophile or base [31]. "Retaining" hydrolases might also act as glycosyl transferase. All lignin-active peroxidases are heme-containing, some with manganese co-active center, and phenol oxidases are copper-containing oxidoreductases, relying on electron-transfer from lignin to high valence $\mathrm{Fe}(\mathrm{V} / \mathrm{VI})$-oxo, $\mathrm{Mn}(\mathrm{III})$, or $\mathrm{Cu}(\mathrm{II})$, which leads to subsequent radicalization, bond scission, or derivatization in lignin [32].

\subsection{Cellulases}

Hydrolytic scission of the $\beta(1 \rightarrow 4)$ glucosidic bond in cellulose, leading to the formation of glucose (Glc) and short cellodextrins, is carried out mainly by cellulases, a group of enzymes comprising cellobiohydrolase ( $\mathrm{CBH})$, endo-1,4- $\beta$-D-glucanase (EG), and $\beta$-glucosidase (BG). Although cellulose is relatively simple in terms of composition (anhydro-Glc units only) and morphology (mainly amorphous and monoclinic $\mathrm{I}_{\beta}$ or triclinic $\mathrm{I}_{\alpha}$ crystalline), there is a vast natural diversity of cellulases with catalytic modules belonging to $\sim 14 \mathrm{GH}$ families to accommodate four major reactions modes and different synergisms (for recently studied examples, see [33-35]). 


\subsubsection{Cellobiohydrolase}

Degradation of crystalline cellulose is carried out mainly by $\mathrm{CBHs}$, thus the enzymes are indispensable for industrial enzymatic lignocellulose degradation. Archetypical CBHs are found in GH6 and 7, as well as 48, families. GH7 CBH is found in all known cellulolytic fungi (based on secretome or genome information). GH6 $\mathrm{CBH}$ is also found in many cellulolytic fungi. Among secreted proteins and enzymes of cellulolytic fungi, up to $70 \%$ wt or so may be CBHs [36-38]. Also known as CBH-I (EC 3.2.1.-), GH7 CBH has specificity towards the reducing end of a cellulose chain. In contrast, GH6 CBH, also known as $\mathrm{CBH}-\mathrm{II}$ (EC 3.2.1.91), can be specific towards the non-reducing end of a cellulose chain. Such "opposing" specificities render GH7 and 6 CBHs highly synergistic and cooperative in degrading their common substrate.

The $\mathrm{CBH}$ catalytic core features tunnel-like active sites, a topology that equips $\mathrm{CBH}$ with the ability to hydrolyze cellulose "processively": it threads into the end of a cellulose chain through its active site, cleaves off a cellobiosyl unit, glides down the chain, and starts the next hydrolysis step [31,39]. A CBM may assist the catalytic core with processivity [40]. Such processive reactions, plus the insolubility of the cellulose substrate, makes CBH kinetics deviant from the Michaelis-Menten model, and show significant fractal and "local jamming" effect [41-43]. Processive CBH movement can be obstructed by kinks or other impediments on the cellulose surface; and as such it has been suggested that $\mathrm{k}_{\text {(off) }}$ values may be a major factor in $\mathrm{CBH}$ efficiency $[44,45]$.

GH7 CBH-I may have approximately ten anhydro-Glc-binding subsites within its active tunnel, in which a cellulose segment or cellodextrin is bound and activated via $\mathrm{H}$-bonding and $\pi$-stacking with key amino acid residues. In addition to the catalytic core, many CBHs also have CBMs, which is believed key in CBH's action on crystalline cellulose.

\subsection{2. endo-1,4- $\beta$-Glucanase}

Degradation of amorphous cellulose can be carried out by EGs (EC 3.2.1.4). Unlike CBH, EG hydrolyzes internal glycosidic bonds in cellulose with a random, on-off fashion. Such dynamics make EG well-suited to less orderly or partially shielded cellulose parts, generating new cellulose chain ends for $\mathrm{CBH}$ action. A few EGs can act "processively" on crystalline cellulose $[13,46]$. There is a significant synergism between $\mathrm{CBH}$ and $\mathrm{EG}$, and their co-presence and cooperation are determinant for highly efficient enzymatic systems of industrial biomass-conversion.

Widely distributed among various organisms, different EGs have a catalytic core belonging to more than ten GH families, of which GH5, 7, 9, 12, 45, and 48 are representative. Typical cellulolytic fungi secrete EGs at $20 \%$ wt level in their secretomes [36-38]. Also known as EG-I, II, III, and V, respectively, $\mathrm{GH} 7,5,12$, and $45 \mathrm{EG}$ are most common in natural fungal cellulase mixes. Most cellulolytic fungi and bacteria produce numerous EGs. Although they all act on the same cellulose substrate, they do so through differing mechanisms ("inverting" for GH6, 9, 45, and 48 EGs; "retaining” for GH5, 7, 12 EGs). Such EG “plurality" may relate to different EGs' side-activities on hemicellulose in degrading complex lignocellulose [47], or synergism between processive and conventional EGs [13]. 
The active sites of most EGs are cleft- or groove-shaped, inside which a cellodextrin or a cellulose segment may be bound and acted on by EG. In addition to the catalytic core, EGs may possess CBMs or other domains. CBMs may direct host EG, but is not a pre-requisite, for EG's action.

\subsection{3. $\beta$-Glucosidases}

Degradation of cellobiose, as well as other cellodextrins, is carried out by BG or cellobiose hydrolase (EC 3.2.1.21). Unlike $\mathrm{CBH}$ and $\mathrm{EG}, \mathrm{BG}$ in general are not modular (lacking distinct CBMs), and have pocket-shaped active sites to act on the non-reducing Glc unit from cellobiose or cellodextrin [48]. BGs belong to the GH1, 3, and 9 families, with GH1 and 3 BGs being archetypical [49]. Unlike the majority of biomass degrading enzymes, the activity of BG, which acts upon soluble rather than insoluble substrate, can be studied using traditional kinetic models [50].

Many cellulolytic fungi produce one or more BGs at levels of about $1 \%$ of total secreted proteins, significantly lower than that of $\mathrm{CBH}$ and EG [36-38]. However, BG plays a key role in the efficiency of an enzymatic lignocellulose-degrading system, because its action on cellobiose mitigates product inhibition on $\mathrm{CBH}$ and EG. For industrial biomass conversion targeting high feedstock loads, supplementing BG to common microbial cellulolytic enzyme preparations can be imperative, because of high cellobiose level during the enzymatic conversion [51].

GH1 BGs tend to be more resilient to Glc (product) inhibition, as well as more active on different di- or oligosaccharides, than GH3 BGs do. Thus having GH1 BG might enable a cellulolytic enzyme system to be more potent in degrading complex lignocellulose.

\subsection{Hemicellulases}

In plant cell walls, cellulose is entangled with and shielded by hemicellulose, a group of complex polysaccharides made by different glyco-units and glycosidic bonds. Degradation of hemicellulose, which not only "liberates" cellulose for cellulases but also converts hemicellulose into valuable saccharides, is carried out mainly by an array of interdependent and synergistic hemicellulases.

Common hemicelluloses include $\beta$-glucan, xylan, xyloglucan, arabinoxylan, mannan, galactomannan, arabinan, galactan, polygalacturonan, etc., which are targets of $\beta$-glucanase, xylanase, xyloglucanase, mannanase, arabinase, galactanase, polygalacturonase, glucuronidase, acetyl xylan esterase, and other enzymes [22,52]. Among hemicellulases, glycoside hydrolases (belonging to about 29 GH families) hydrolyze glycosidic bonds, carbohydrate esterases (belonging to about 9 CE families) hydrolyze ester bonds, polysaccharide lyases (belonging to about 5 PL families) cleave glycosidic bonds. endo-Hemicellulases cleave internal/backbone glycosidic bonds, whereas other glycosidases remove mainly the chain's substituents or side chains. Cellulolytic microbes produce many hemicellulases along with cellulases for effective lignocellulose degradation (for recently studied examples see [36-38]).

Different plants have different hemicelluloses: acetylated (galacto)glucomannan (as well as arabinoglucuronoxylan), glucuronoxylan, and arabinoxylan are major hemicellulose in softwood, hardwood, and grass, respectively [52]. Hence different hemicellulase combinations are needed for different biomass feedstocks in industrial biomass conversion. Synergism of hemicellulases is found both amongst hemicellulases themselves and between hemicellulases and cellulases [2,35,53-56]. 


\subsection{1. endo- $\beta$-Xylanases and $\beta$-Xylosidase}

Degradation of (glucurono)(arabino)xylan, a group of $\beta(1 \rightarrow 4)$ linked D-xylopyranosyl (Xyl) polysaccharides with different O-substitutions by acetyl, glucuronoyl (GlcU), arabinosyl (Ara), or other substituents, is mainly carried out by endo-xylanase (EX, EC 3.2.1.8), which hydrolyzes backbone glycosidic bonds in xylan. Widely distributed among archaea, bacteria, fungi, and plants, EXs have catalytic cores belonging to the GH8, 10, 11, 30, and 43 families, with GH10 and GH11 EX being archetypical [57]. GH10 and 11 EX differ in substrate specificity: GH10 EX produces shorter oligosaccharides and has more activity on substituted xylan [58]. Besides the catalytic core, one or more CBMs or other domains may be found in EXs [59].

As BG does for EG, $\beta$-xylosidases (BX, EC 3.2.1.37) hydrolyze xylobiose or other xylooligosaccharides, after their production from xylan by EX [60]. BXs have catalytic cores belonging to the $\mathrm{GH} 3,30,39,43,52$, and 54 families. Many BXs have $\alpha$-L-arabinofuranosidase activity. Like cellulases, xylanases also employ either an "inverting" or a "retaining" mechanism based on a nucleophile and a general acid catalytic diad for their catalysis.

Among enzymes secreted by cellulolytic fungi, xylanases often account for $<1 \% \mathrm{wt}$, although multiple xylanases may be produced [36-38]. Xylanases may provide, or benefit from, significant synergism among themselves, with other hemicellulases, or with cellulases: GH11 EX may produce large xylooligosaccharides for GH10 EX action, debranching hemicellulases may remove substituents to facilitate EX reaction, or EX may degrade xylan in lignocellulose to expose cellulose for cellulase reaction [61]. Hence, optimizing a lignocellulose-degrading enzyme mix with xylanases can be key for industrial biomass conversion, especially for hardwood or grass-based feedstocks enriched in (arabion)xylan.

\subsubsection{Acetyl Xylan Esterase, Feruloyl Esterase, and Glucuronoyl Esterase}

Acetyl, feruloyl (or other hydroxycinnamoyls), and GlcU groups are common ester substituents in xylan or other hemicelluloses. Their removal, often key for effective EX or other endo-hemicellulases activity, is carried out by acetyl xylan esterase (AXE, EC 3.1.1.72), feruloyl esterase (FAE, EC 3.1.1.73), and glucuronoyl esterase (GE, EC 3.1.1.-), respectively. Belonging to CE1, 2, 3, 4, 5, 6, 7, and 12 families, AXE deacetylates substituted $\mathrm{O} 2$ or $\mathrm{O} 3$ sites of backbone glycosyl units in xylan or other hemicelluloses [62]. Belonging to CE1 family, FAE hydrolyzes feruloyl esters at $\alpha$-L-Ara (O2 or O5 site), $\beta$-D-galactosyl (Gal, O6 site), or $\alpha$-D-Xyl side chains of arabinan/arabinoxylan, rhamnogalacturonan, or xyloglucan [63]. Belonging to CE15 family, GE demethylates O6-methyl glucuronoyl (GlcU) $\alpha(1 \rightarrow 2)$ linked to backbone Xyl in glucuronoarabinoxylan [64]. These esterases have the canonical Ser-His-Asp catalytic triad found in other esterases, lipases, or serine proteases.

FAEs may have CBMs together with their catalytic core. Different FAEs have different specificity towards different hydroxycinnamoyl ester bonds, which are involved in linking hemicellulose to lignin [65]. Different AXEs, FAEs, and GEs may cooperate in attacking complex hemicellulose. AXE or FAE can assist endo-hemicellulases by deacetylating, deferulating, or delignifying hemicelluloses. GEs can assist $\alpha$-glucuronidase by hydrolyzing GlcU ester. For industrial biomass conversion, 
supplementing AXE or FAE may enhance endo-hemicellulases' activity, by attacking acetylated hardwood xylan or ferulated grass arabinoxylan.

\subsection{3. $\alpha$-L-Arabinofuranosidase, $\alpha$-Galactosidase and $\alpha$-Glucuronidase}

Removal of Ara substituent is carried out by $\alpha$-L-arabinofuranosidase (AF, EC 3.2.1.55), a group of enzymes whose catalytic cores belong to GH3, 43, 51, 54, and 62 families [66]. Many AFs contain CBMs. There are specificity differences among AFs: some prefer single Ara esterifying either O2, 3, or 5 site, while others prefer dual Ara esterifying $\mathrm{O} 2$ and 3 sites.

Removal of Gal substituent linked via $\alpha$-glycosidic bonds to galactomannan, pectin, or other hemicelluloses is carried out by $\alpha$-galactosidase (EC 3.2.1.22), a group of enzymes whose catalytic cores belong to $\mathrm{GH} 4,27,36,57$, and 110 families. GH4 and $110 \alpha$-galactosidases rely on $\mathrm{NAD}^{+}$ cofactor and a redox mechanism to hydrolyze their substrates, unique among known glycosidases [67].

Removal of $\alpha(1 \rightarrow 2)$ linked glucuronoyl or its methyl ester in xylan (often at the $\mathrm{O} 2$ site) or other hemicelluloses is carried out by $\alpha$-glucuronidases (AG, EC 3.2.1.139), a group of enzymes whose catalytic cores belong to GH67 and 115 families [68,69]. Some AGs have higher specificity to glucuronated xylooligosaccharides, while others have higher specificity to polymeric glucuronoxylan [61].

AF, $\alpha$-galactosidase, and $\alpha$-glucuronidases may assist xylanase, pectinase, or other hemicellulases by debranching their polymeric substrates. Supplementing AF to lignocellulose-degrading enzyme mix may be highly beneficial when softwood (abundant in arabinoglucuronoxylan) or grass (rich in arabinoxylan) feedstocks are targeted.

\subsubsection{Glucanase, Mannanase, Xyloglucan Hydrolase and Pectinase}

Degradation of $\beta(1 \rightarrow 3)$, $(1 \rightarrow 4)$, or $(1 \rightarrow 6)$ glucan can be carried out by (non-EG or BG) $\beta$-glucanases (EC 3.2.1.-), a diverse group of endo- or exo-acting, glycosidic bond type-specific or promiscuous enzymes whose catalytic cores belong to GH3, 5, 12, 16, 17, 55, 64, and 81 families [70]. Many cellulolytic microbes secrete, or anchor in cell membrane, one or more $\beta$-glucanases, whose differential specificities may enable cooperative degradation of complex $\beta$-glucans heterogeneous in backbone architecture or glycosidic bonds.

Degradation of (galacto)(gluco)mannans, $\beta(1 \rightarrow 4)$-D-mannosyl or manno/glucopyranosyl polymers with variable $\alpha(1 \rightarrow 6) \mathrm{D}$-Gal side chain as well as $\mathrm{O} 2$ and/or O3 acetylation, can be carried out by mannanase (EC 3.2.1.78), a group of widely distributed, hydrolytic enzymes with catalytic cores belonging to GH5, 26, and 113 families [71]. Mannooligosaccharides produced by mannanase can be further degraded by $\beta$-mannosidases (EC 3.2.1.25), whose catalytic cores belong to GH1, 2, and 5 families. In addition to a catalytic core, mannanases may possess one or more CBM (specific to mannan or cellulose) or other domains (e.g., Ig-like and S-layer module). Many cellulolytic microbes co-secrete mannanases with cellulases, xylanases, and other enzymes [36]. Feedstocks abundant in (galacto)glucomannan, such as softwood, are likely in need of enzyme mixes containing sufficient mannanase activity for effective substrate degradation/conversion.

Degradation of xyloglucan, $\beta(1 \rightarrow 4)$ glucan with $\alpha(1 \rightarrow 6)$ linked Xyl substituted by either $\alpha(1 \rightarrow 2)$ L-Ara or $\beta(1 \rightarrow 2)$ D-Gal units (partially acetylated or substituted by $\alpha(1 \rightarrow 2)$ L-fucopyranosyl (Fuc)), can be carried out by xyloglucan hydrolases (EC 3.2.1.150, 151, 155), whose catalytic cores belong to 
GH5, 12, 16, 44, and 74 families, and that are part of the xyloglucan transferase/hydrolase (XTH) superfamily [72]. Many xyloglucan hydrolases have minor EG activity, while many EGs have minor xyloglucan-hydrolyzing activity [47]. AFs and esterases may de-branch xyloglucan, thus allowing more effective xyloglucan hydrolase activity. Xyloglucan hydrolases may be important for industrial biomass conversion, because the degradation of xyloglucan could enhance cellulase accessibility to cellulose [73].

A diverse group of pectinolytic enzymes are responsible for the degradation of pectic polysaccharides, consisted of $\alpha(1 \rightarrow 4)$ poly- $\alpha$-(rhamno)galacturonic acids with variable backbone methylation/acetylation and Ara and Gal side chains branching [74,75]. Common pectinolytic enzymes include polygalacturonases (EC 3.2.1.15, 67, 82) with catalytic cores belonging to GH28 family, pectin/pectate lyases (EC 4.2.2.2, 6, 9, 10) with catalytic cores belonging to PL1, 2, 3, 9, and 10 families, and pectin methyl esterase (EC 3.1.1.11) with catalytic cores belonging to CE8 family. Unlike hydrolytic polygalacturonases, pectin/pectate lyases cleave an O-C4 glycosidic bond, assisted by $\mathrm{C} 6$-uronate, to form a $\Delta 4: 5 \mathrm{C}=\mathrm{C}$ bond at the non-reducing side of galacturonoyl unit. Many pectinolytic hydrolases and lyases act on both pectin and pectate. Different pectinolytic enzymes act in concert: endo- and exo-enzymes synergize each other as EG and $\mathrm{CBH}$ do; hydrolases and lyases may act on different parts of pectin/pectate; and methyl esterases demethylate pectin to help pectate-specific enzymes. AF, galactosidase, and other enzymes may enhance pectinolytic enzymes' action by removing the side chains from polyrhamnogalacturonan. Rich in pectic polysaccharides, sugar beet pulp and fruit residue-based feedstocks are likely in need of sufficient pectinolytic enzymes for effective enzymatic degradation/conversion.

\subsection{Lignocellulose Oxidoreductases}

The secretomes of most cellulolytic microbes (particularly white and brown rots) contain oxidoreductases, in some cases at quite significant levels, whose co-presence with hydrolytic or lytic enzymes indicates the importance of having an oxidoreductive system as part of effective biological lignocellulose degradation $[5,32,37,76,77]$. The main task of these oxidoreductases is likely aimed at degradation of lignin, a highly heterogeneous and recalcitrant aromatic polymer (consisted of various syringyl, guaiacyl, or other hydroxyphenyl units) entangled with hemicellulose or cellulose and inactive/inhibitory to (hemi)cellulases. Lignin degradation is imperative for industrial enzymatic biomass-conversion, because it not only increases (hemi)cellulose accessibility for (hemi)cellulase but also diminishes (hemi)cellulase inactivation caused by lignin adsorption.

Lignin peroxidase (EC 1.11.1.14), Mn peroxidase (EC 1.11.1.13), and versatile peroxidase (EC 1.11.1.16) are extracellular fungal heme peroxidases (belonging to LO2 family) with high potency to oxidatively degrade lignin. Upon interaction with $\mathrm{H}_{2} \mathrm{O}_{2}$, these enzymes form highly reactive $\mathrm{Fe}(\mathrm{V})$ or Fe(IV)-oxo species, which abstract electrons from lignin (to cause oxidation or radicalization) either directly or via $\mathrm{Mn}$ (III) species. Laccase (EC 1.10.3.2) is a multi-copper oxidase (belonging to LO1 family) secreted by numerous lignocellulolytic fungi. This enzyme can directly oxidize phenolic parts of lignin, or indirectly oxidize non-phenolic lignin parts with the aid of suitable redox-active mediator.

Aryl-alcohol oxidase (EC 1.1.3.7), glyoxal oxidase (EC 1.1.3.-), and various carbohydrate oxidases (EC 1.1.3.4, 9, 10) are also involved in natural lignocellulose degradation. These enzymes, belonging 
to LDA1-6 families, can generate $\mathrm{H}_{2} \mathrm{O}_{2}$ from $\mathrm{O}_{2}$, with concomitant oxidation of aromatic alcohol, glyoxal, or reducing carbohydrates. The generated $\mathrm{H}_{2} \mathrm{O}_{2}$ may support lignin-degrading peroxidases or power Fenten-type chemistry that degrades lignin non-enzymatically.

Cellobiose dehydrogenase (EC 1.1.99.18) is produced by many lignocellulotic fungi. The flavoheme enzyme (belonging to LO3 family) may dehydrogenate or oxidize cellobiose or other cellodextrins to corresponding aldonolactones, with concomitant quinone reduction to phenol or $\mathrm{O}_{2}$ reduction to $\mathrm{H}_{2} \mathrm{O}_{2}$. Until recently, the role of $\mathrm{CDH}$ in enzymatic degradation of lignocellulose was largely considered only from a Fenton chemistry perspective [78] or mitigation of cellulases' product inhibition by cellobiose [79]; however, the recent discovery of the CDH stimulation of GH61 enzyme activity may shed new light upon its function [80-82] (see Section 3.1).

The lignin-degrading oxidative species generated by these oxidoreductases may attack inhibitors of industrial sugar-to-fuel/chemical microbial conversion, making the process more effective [83]. However, they may also attack (hemi)cellulose or even (hemi)cellulases, potentially hampering industrial enzymatic (hemi)cellulose conversion [84,85]. For the peroxidases, autooxidation or inactivation may severely limit their performance. Applying such enzymes to industrial enzymatic biomass conversion needs further research for overall benefit.

\section{Emergent Industrial Lignocellulose-Degrading Enzymes}

In addition to the enzyme categories above, lignocellulose degrading microbes encode and secrete a number of proteins, often co-induced and secreted with canonical (hemi)cellulases, with more enigmatic functionalities. Some of these molecules, when added to cellulases, result in large increases in lignocellulose breakdown, while others display less dramatic stimulatory effects on cellullase activity (at least under laboratory conditions). An improved understanding to how these enzymes function may result in significant improvements in industrial lignocellulose degradation, and as such, much attention is currently focused on these molecules.

\subsection{Discovery of GH61 Cellulase-Enhancing Protein}

Many cellulolytic fungi encode and express proteins which are classified as members of the Glycoside Hydrolase 61 (GH61) family [36-38], but due to the unusual characteristics of the GH61s, it took more than a decade after the initial discovery of this group of enzymes to begin to understand their function. The first of these proteins was found during a cDNA screen for the Trichoderma reesei complement to the Saccharomyces cerevisiae gene SEC1, which is involved in secretion. Unexpectedly, several of the isolated $T$. reesei cDNA clones, which were shown to suppress the temperature sensitive secl-1 mutant of $S$. cerevisiae, contained a consensus sequence for a CBM. Based on this CBM homology, the protein encoded by this cDNA was expressed and tested for cellulase activity. The prepared protein sample was observed to have weak activity in the breakdown of $\beta$-glucan, carboxymethyl cellulose (CMC), and phosphoric acid-swollen cellulose (PASC), and was classified as T. reesei EG-IV [86]. A further characterization of this protein reported that this enzyme could cause the release of small amounts of soluble cellooligosaccharides from cellulosic substrates [87]. Similar results, showing weak if any EG activity, were reported for a homologous GH61 encoded by Aspergillus kawachii [88]. 
Suspicion that GH61 was not an authentic EG got strong support from GH61 crystal structures. EGs usually have a cleft in which the cellulose strand binds to the enzyme, typically through interactions with aromatic residues, and a hydrolase active site near this cleft containing a catalytic glutamic or aspartic acid. The structure of T. reesei GH61B [89] contains neither a cleft nor an obvious hydrolase active site, but rather displays a surface-exposed divalent metal binding site surrounded by hydrophobic residues, suggesting a possible carbohydrate binding surface and active site of unknown function. Structurally, T. reesei GH61B is homologous to chitin binding protein 21 (CBP21, belonging to CBM33 family) from the bacterium Serratia marcescens, a molecule which at the time was thought to be a non-catalytic protein which enhanced chitin breakdown by chitin hydrolases [90]. Another publication reported the structure of Thielevia terrestris GH61E, which also includes a surface exposed metal binding site; this report also included significant biochemical analysis of T. terrestris GH61E, T. terrestris GH61B, and Thermoascus aurantiocus GH61A activity upon both model and industrial cellulosic substrates [91]. In this analysis, the GH61 molecules tested displayed only negligible ability to cleave any of the cellulosic or hemicellulosic substrates tested. However, addition of GH61s to Trichoderma cellulase cocktails was shown to greatly enhance the activity of these cellulases in lignocellulose degradation, lowering the required enzyme concentration for substrate breakdown by a factor of two. Confirming the role of metal in GH61 activity, the GH61 cellulase-boosting effect was inhibited by addition of EDTA, and metal binding site mutants of GH61 displayed greatly reduced or absent cellulase boosting activity. Surprisingly, the addition of GH61 has no stimulatory effect on Trichoderma cellulases' breakdown of "pure" cellulose substrates (e.g., microcrystalline cellulose) suggesting that a non-cellulose component might be a requirement for or the target of GH61 activity [91]. While the industrial significance of GH61 as a critical component in cellulase mixtures was established, the biochemical activity of these molecules remained mysterious.

Recently, the functions of members of the fungal GH61 family, and members of the related bacterial CBM33 family, were revealed. Several of these enzymes have been shown to act by cleaving cellulose [80-82,92-95] or the structurally related polysaccacharide chitin [96]; and while some differences in data and analysis exist amongst these publications it is clear that GH61 and CBM33 enzymes do not function as typical glycoside hydrolases.

Unlike canonical glycoside hydrolases, GH61 and CBM33 enzymes require a redox-active factor for activity. This cofactor requirement explains the formerly puzzling results which show little or no GH61 activity upon model cellulose substrates, but a substantial increase in complex biomass degradation by Trichoderma cellulases or mixtures upon GH61 supplementation [91]; when the soluble phase of dilute acid pretreated biomass was added to model cellulose substrates, GH61 was shown to act upon those substrates, indicating that requisite GH61 cofactor was present in soluble (and perhaps also in insoluble) fractions of pretreated biomass [94]. GH61 and CBM33 polysaccharide cleaving-activity has been demonstrated upon addition of a small molecule reducing-agent such as ascorbate, glutathione, or gallate [92-96] or the fungal enzyme cellobiose dehydrogenase [80-82]. Analysis of the polysaccharide cleavage products formed by GH61 and CBM33 molecules reveals that these enzymes release oxidized cellooligosaccharides, though significant quantities of non-oxidized oligosaccharides are also detected in some studies [93-95]. Unlike other glycoside hydrolases, which are more active on cellooligosaccharides than crystalline cellulose, GH61 and CBM33 enzymes appear inactive upon cellooligosaccahrides [81,93-96]. The position of the oxidation on the oligosaccharide 
products has been reported on the reducing end [81,82,93,95], non-reducing end [81,82], or both [94], which could suggest differences amongst these enzymes. GH61 proteins also require a metal for this cellulose cleaving activity, specifically copper which binds tightly to the protein in a type-2 copper site geometry [82,94,96]. Interestingly, despite the large potential of this enzyme class to promote lignocellulose breakdown, the precise cleavage mechanism of GH61 and CBM33 enzymes is unclear, and is the subject of ongoing study by multiple groups.

\subsection{Expansin, Swollenin, and Loosinin}

Expansins are a class of plant proteins which interact with and modify cell walls and/or cell wall components by an unknown activity, thought to result in expansion, slippage, or lengthening of cell wall structures. These two-domain proteins consist of a domain homologous to the GH45 EG catalytic core and a second domain homologous to Group II grass pollen allergens; both domains have no known catalytic function and display no detectable hydrolytic activity on lignocellulosic or model substrates (expansins are thoroughly reviewed by [97]). T. reesei has been shown to express a protein, named swollenin, which has sequence homology to plant expansins and displays a similar mysterious disruptive effect on cellulosic substrates. Isolated T. reesei swollenin has been shown, without cause to formation of detectable reducing ends, to weaken filter paper and to affect superstructural changes in cotton fibrils by light and atomic force microscopy [98].

Despite the apparent lack of direct lytic activity on cellulose, the addition of $T$. reesei swollenin significantly increases the breakdown of filter paper by cellulases [99,100]. Swollenin from Aspergillus fumigatus is reported to have weak lytic activity on CMC but no apparent hydrolytic activity on microcrystalline cellulose, though treatment of microcrystalline cellulose with A. fumigatus swollenin reduced apparent microcrystalline cellulose particle size and potentiated breakdown of the cellulose by hydrolytic cellulases [101]. The basidiomycete Bjerkandera adusta produces a similar protein, loosinin, which increases cellulase activity on cotton and agave bagasse [102]. Bacterial species, including Bacillus subtilis [103] and Hahella chejuensis [104], also produce expansin-like molecules. Like fungal swollenins, bacterial expansins alter cellulose fiber structure and promote the breakdown of cellulose by hydrolases without showing detectable direct hydrolase activity [104,105]. Recent structural and mutational studies of expansin EXLX1 from B. substilis have shed some light on these proteins, demonstrating that several clustered residues on GH45-like domain are required for EXLX1's cell wall modifying activity, and that the second domain is likely a new type of CBM $[103,106]$. Further study will be required in order to determine the mechanism by which these molecules increase lignocellulose conversion and allow industrial exploitation of this class of protein.

\subsection{CIP Proteins}

CIP1 and CIP2 (cellulose induced protein-1 and -2, respectively) were first found in a transcriptional analysis of $T$. reesei. Both contain a CBM and are co-regulated with known cellulases [107]. The function of CIP1 is unknown, though it is claimed that CIP1 from T. reesei has weak activity on $p$-nitrophenyl $\beta$-D-cellobioside [108] and some synergistic activity with both GH61 and swollenin [109]. CIP2, found in both T. reesei and Schizophyllum commune, has recently been shown to be an esterase that cleaves the methyl ester of 4- $O$-methyl-D-glucuronic acid [110]. This enzyme, now classified as 
the first member of CE15 family (EC 3.1.1.-), likely acts in the cleavage of hemicellulose-lignin crosslinks. Further investigation of both the functions and the potential of these enzymes in industrial applications are needed.

\subsection{Cellulosomes}

While many microorganisms secrete biomass degrading enzymes into their environment, other microbes, particularly anaerobic biomass degrading microbes, use cell-surface linked enzymes to break down lignocellulosic materials. Cellulosomes are arrays of multiple cellulase and hemicellulase proteins, assembled by specific interactions between dockerin domains on the enzyme and cohesins bound to structural scaffoldins on the microbial surface (reviewed extensively in [28-30,111]). This spatial clustering of multiple lignocellulose degrading enzymes results in an increased synergy between lytic activities $[28,30,112]$. It has been shown that recombinant cellulosomes can be transplanted to other industrially useful organisms, such as S. cerevisiae [113,114] and B. subtilis [115]. The ability of cellulosomes to cluster activities may present unique capabilities, both in synergistic breakdown of a substrate and in targeted degradation of specific biomass components.

\section{Perspectives}

Microbial breakdown of lignocellulosic biomass is a highly complex process, requiring multiple types of synergistic catalytic activities simultaneously acting upon a variety of both soluble and insoluble polymeric substrates. Industrial biomass utilization processes will also require a combination of activities in order to degrade lignocellulosic feedstocks, but with the additional complication that thermal and chemical pretreatment processes, which improve physical substrate access to the enzymes, alter the substrate chemically and form or solubilize inhibitory compounds that can negatively impact enzyme performance [116]. When combined with traditional cellulases, previously uncharacterized enzymes, some of which have been discussed in this review, have a high degree of synergy in the degradation of pretreated lignocellulosic substrates [91]. Further improvements in and synergies with industrial processes are possible with other emergent enzyme families, and with yet to be discovered or currently overlooked enzyme activities. The exploration of biological diversity, particularly from extremophilic organisms, may reveal new cellulases with improved properties for specific industrial processes. For example, it has been shown that cellulases from halophilic organisms have higher resistance to inactivating ionic liquid residues from certain pretreatments processes $[117,118]$. Protein engineering of enzymes, such as components of the $T$. reesei cellulase complex, to improve their suitability for industrial biomass applications has been a growing area of activity within both academic and industrial research into biomass conversion. Many groups report improvements to the properties of biomass degrading enzymes, such as increased thermostability or thermoactivity, altered $\mathrm{pH}$ optima, decreased lignin binding or glucose inhibition, or improved activity on crystalline cellulose.

This review has focused on specific enzymes and activities. However, the production of these enzymes, or more likely mixtures of enzymes, must also be considered in order to develop viable enzymatic biomass conversion technologies. An enzyme mixture with improved activity but which is prohibitively expensive to produce on a large scale will be of little industrial use. One topic of great consequence, but which is beyond the scope of this review, is the development of production hosts for 
biomass degrading enzymes. Significant efforts have been made, by either natural diversity exploration, classical mutagenesis, or genetic engineering, which have resulted in strains with improved enzyme expression profiles, higher protein secretion levels, or the ability to utilize pretreated biomass as a nutrient source. However, the required activities, or ratios of activities, which will be required for optimal breakdown of lignocellulose will vary among different types of pretreatment and biomass, making the ability to design strains, and the enzymes they express, optimized for a specific process or robust for many processes, an area of mounting importance.

While the enzymatic breakdown of lignocellulosic biomass is a complicated process, involving many activities which work in tandem to decompose a heterogeneous and recalcitrant substrate, the understanding of these enzymes and activities has increased significantly in recent years. Ongoing studies of both known and yet-to be discovered enzymes will provide further insight into this complex process and give guidance as to how enzymes can be better applied to a variety of industrial processes.

\section{Acknowledgement}

We thank Robert L. Starnes of Novozymes for critical reading of this manuscript.

\section{References}

1. Lynd, L.R.; Laser, M.S.; Bransby, D.; Dale, B.E.; Davison, B.; Hamilton, R.; Himmel, M.; Keller, M.; McMillan, J.D.; Sheehan, J.; Wyman, C.E. How biotech can transform biofuels. Nat. Biotechnol. 2008, 26, 169-172.

2. Kumar, R.; Singh, S., Singh, O.V. Bioconversion of lignocellulosic biomass: biochemical and molecular perspectives. J. Ind. Microbiol. Biotechnol. 2008, 35, 377-391.

3. Wackett, L.P. Biomass to fuels via microbial transformations. Curr. Opin. Chem. Biol. 2008, 12, 187-193.

4. Margeot, A.; Hahn-Hagerdal, B.; Edlund, M.; Slade, R.; Monot, F. New improvements for lignocellulosic ethanol. Curr. Opin. Biotechnol. 2009, 20, 372-380.

5. Dashtban, M.; Schraft, H.; Qin, W. Fungal bioconversion of lignocellulosic residues; opportunities \& perspectives. Int. J. Biol. Sci. 2009, 5, 578-595.

6. Sánchez, C. Lignocellulosic residues: biodegradation and bioconversion by fungi. Biotechnol. Adv. 2009, 27, 185-194.

7. $\mathrm{Xu}, \mathrm{F}$. Biomass-converting enzymes and their bioenergy applications. In The Manual of Industrial Microbiology and Biotechnology, 3rd ed.; Baltz, R.H., Demain, A.L., Davies, J.E., Bull, A.T., Junker, B., Katz, L., Lynd, L.R., Masurekar, P., Reeves, C.D., Zhao, H., Eds.; American Society for Microbiology Press: Washington, DC, USA, 2010; pp. 495-508.

8. Girio, F.M.; Fonseca, C.; Carvalheiro, F.; Duarte, L.C.; Marques, S.; Bogel-Lukasik, R. Hemicelluloses for fuel ethanol: A review. Bioresour. Technol. 2010, 101, 4775-4800.

9. Sims, R.E.H.; Mabee, W.; Saddler, J.N.; Taylor, M. An overview of second generation biofuel technologies. Bioresour. Technol. 2010, 101, 1570-1580.

10. Chandel, A.K.; Singh, O.V. Weedy lignocellulosic feedstock and microbial metabolic engineering: advancing the generation of "Biofuel". Appl. Microbiol. Biotechnol. 2011, 89, 1289-1303. 
11. Zhang, Y.H.P. What is vital (and not vital) to advance economically-competitive biofuels production. Process Biochem. 2011, 46, 2091-2110.

12. Henrissat, B.; Sulzenbacher, G.; Bourne, Y. Glycosyltransferases, glycoside hydrolases: surprise, surprise! Curr. Opin. Struct. Biol. 2008, 18, 527-533.

13. Wilson, D.B. Three microbial strategies for plant cell wall degradation. Ann. N. Y. Acad. Sci. 2008, 1125, 289-297.

14. Wilson, D.B. Cellulases and biofuels. Curr. Opin. Biotechnol. 2009, 20, 295-299.

15. Baldrian, P.; Valásková, V. Degradation of cellulose by basidiomycetous fungi. FEMS Microbiol. Rev. 2008, 32, 501-521.

16. Blumer-Schuette, S.E.; Kataeva, I.; Westpheling, J.; Adams, M.W.; Kelly, R.M. Extremely thermophilic microorganisms for biomass conversion: status and prospects. Curr. Opin. Biotechnol. 2008, 19, 210-217.

17. Gowen, C.M.; Fong, S.S. Exploring biodiversity for cellulosic biofuel production. Chem. Biodivers. 2010, 7, 1086-1097.

18. Jovanovic, I.; Magnuson, J.K.; Collart, F.; Robbertse, B.; Adney, W.S.; Himmel, M.E.; Baker, S.E. Fungal glycoside hydrolases for saccharification of lignocellulose: outlook for new discoveries fueled by genomics and functional studies. Cellulose 2009, 16, 687-697.

19. Yeoman, C.J.; Han, Y.; Dodd, D.; Schroeder, C.M.; Mackie, R.I.; Cann, I.K.O. Thermostable enzymes as biocatalysts in the biofuel industry. Adv. Appl. Microbiol. 2010, 70, 1-55.

20. De Vries, R.P.; Battaglia, E.; Coutinho, P.M.; Henrissat, B.; Visser, J. (Hemi-)cellulose degrading enzymes and their encoding genes from Aspergillus and Trichoderma. In The Mycota X. Industrial Applications, 2nd ed.; Hofrichter, M., Ed.; Springer-Verlag: Berlin, Germany, 2010; Chapter 16.

21. Gilbert, H.J. The biochemistry and structural biology of plant cell wall deconstruction. Plant Physiol. 2010, 153, 444-455.

22. Van den Brink, J.; de Vries, R.P. Fungal enzyme sets for plant polysaccharide degradation. Appl. Microbiol. Biotechnol. 2011, 91, 1477-1492.

23. Enzyme Nomenclature; Webb, E.C., Ed.; Academic Press: San Diego, CA, USA, 1992.

24. Cantarel, B.L.; Coutinho, P.M.; Rancurel, C.; Bernard, T.; Lombard, V.; Henrissat, B. The Carbohydrate-Active EnZymes database (CAZy): An expert resource for glycogenomics. Nucleic Acids Res. 2009, 37, D233-D238.

25. Levasseur, A.; Piumi, F.; Coutinho, P.M.; Rancurel, C.; Asther, M.; Delattre, M.; Henrissat, B.; Pontarotti, P.; Asther, M.; Record, E. FOLy: An integrated database for the classification and functional annotation of fungal oxidoreductases potentially involved in the degradation of lignin and related aromatic compounds. Fungal Genet. Biol. 2008, 45, 638-645.

26. Guillen, D.; Sanchez, S.; Rodriguez-Sanoja, R. Carbohydrate-binding domains: Multiplicity of biological roles. Appl. Microbiol. Biotechnol. 2010, 85, 1241-1249.

27. Moser, F.; Irwin, D.; Chen, S.L.; Wilson, D.B. Regulation and characterization of Thermobifida fusca carbohydrate-binding module proteins E7 and E8. Biotechnol. Bioeng. 2008, 100, 1066-1077. 
28. Ding, S.Y.; Xu, Q.; Crowley, M.; Zeng, Y.; Nimlos, M.; Lamed, R.; Bayer, E.A.; Himmel, M.E. A biophysical perspective on the cellulosome: new opportunities for biomass conversion. Curr. Opin. Biotechnol. 2008, 19, 218-227.

29. Gilbert, H.J. Cellulosomes: microbial nanomachines that display plasticity in quaternary structure. Mol. Microbiol. 2007, 63, 1568-1576.

30. Bayer, E.A.; Lamed, R.; Himmel, M.E. The potential of cellulases and cellulosomes for cellulosic waste management. Curr. Opin. Biotechnol. 2007, 18, 237-245.

31. Vocadlo, D.J.; Davies, G.J. Mechanistic insights into glycosidase chemistry. Curr. Opin. Chem. Biol. 2008, 12, 539-555.

32. Martínez, A.T.; Speranza, M.; Ruiz-Dueñas, F.J.; Ferreira, P.; Camarero, S.; Guillén, F.; Martínez, M.J.; Gutiérrez, A.; del Río, J.C. Biodegradation of lignocellulosics: microbial, chemical, and enzymatic aspects of the fungal attack of lignin. Int. Microbiol. 2005, 8, 195-204.

33. Duncan, S.M.; Schilling, J.S. Carbohydrate-hydrolyzing enzyme ratios during fungal degradation of woody and non-woody lignocellulose substrates. Enzym. Microb. Technol. 2010, 47, 363-371.

34. Zhang, M.; Su, R.; Qi, W.; He, Z. Enhanced enzymatic hydrolysis of lignocellulose by optimizing enzyme complexes. Appl. Biochem. Biotechnol. 2010, 160, 1407-1414.

35. Banerjee, G.; Car, S.; Scott-Craig, J.S.; Borrusch, M.S.; Walton, J.D. Rapid optimization of enzyme mixtures for deconstruction of diverse pretreatment/biomass feedstock combinations. Biotechnol. Biofuels 2010, 3, 22.

36. Herpoël-Gimbert, I.; Margeot, A.; Dolla, A.; Jan, G.; Mollé, D.; Lignon, S.; Mathis, H.; Sigoillot, J.-C.; Monot, F.; Asther, M. Comparative secretome analyses of two Trichoderma reesei Rut-C30 and CL847 hypersecretory strains. Biotechnol. Biofuels 2008, 1, 18.

37. Sipos, B.; Benko, Z.; Dienes, D.; Reczey, K.; Viikari, L.; Siika-aho, M. Characterisation of specific activities and hydrolytic properties of cell-wall-degrading enzymes produced by Trichoderma reesei Rut C30 on different carbon sources. Appl. Biochem. Biotechnol. 2010, 161, 347-364.

38. Chundawat, S.P.S.; Lipton, M.S.; Purvine, S.O.; Uppugundla, N.; Gao, D.; Balan, V.; Dale, B.E. Proteomics-based compositional analysis of complex cellulase-hemicellulase mixtures. J. Proteome Res. 2011, 10, 4365-4372.

39. Liu, Y.S.; Baker, J.O.; Zeng, Y.; Himmel, M.E.; Haas, T.; Ding, S.Y. Cellobiohydrolase hydrolyzes crystalline cellulose on hydrophobic faces. J. Biol. Chem. 2011, 286, 11195-11201.

40. Beckham, G.T.; Matthews, J.F.; Bomble, Y.J.; Bu, L.; Adney, W.S.; Himmel, M.E.; Nimlos, M.R.; Crowley, M.F. Identification of amino acids responsible for processivity in a Family 1 carbohydrate-binding module from a fungal cellulase. J. Phys. Chem. B 2010, 114, 1447-1453.

41. Xu, F.; Ding, H. A new kinetic model for heterogeneous (or spatially confined) enzymatic catalysis: Contributions from the fractal and jamming (overcrowding) effects. Appl. Catal. A Gen. 2007, 317, 70-81.

42. Igarashi, K.; Uchihashi, T.; Koivula, A.; Wada, M.; Kimura, S.; Okamoto, T.; Penttilä, M.; Ando, T.; Samejima, M. Traffic jams reduce hydrolytic efficiency of cellulase on cellulose surface. Science 2011, 333, 1279-1282.

43. Warden, A.C.; Little, B.A.; Haritos, V.S. A cellular automaton model of crystalline cellulose hydrolysis by cellulases. Biotechnol. Biofuels 2011, 4, 39. 
44. Kurasin, M.; Väljamäe, P. Processivity of cellobiohydrolases is limited by the substrate. J. Biol. Chem. 2011, 286, 169-177.

45. Praestgaard, E.; Elmerdahl, J.; Murphy, L.; Nymand, S.; McFarland, K.C.; Borch, K.; Westh, P. A kinetic model for the burst phase of processive cellulases. FEBS J. 2011, 278, 1547-1560.

46. Li, Y.; Irwin, D.C.; Wilson, D.B. Increased crystalline cellulose activity via combinations of amino acid changes in the family 9 catalytic domain and family $3 c$ cellulose binding module of Thermobifida fusca Cel9A. Appl. Environ. Microbiol. 2010, 76, 2582-2588.

47. Vlasenko, E.; Schülein, M.; Cherry, J.; Xu, F. Substrate specificity of family 5, 6, 7, 9, 12, and 45 endoglucanases. Bioresour. Technol. 2010, 101, 2405-2411.

48. Langston, J.; Sheehy, N.; Xu, F. Substrate specificity of Aspergillus oryzae family $3 \beta$-glucosidase. Biochim. Biophys. Acta 2006, 1764, 972-978.

49. Eyzaguirre, J.; Hidalgo, M.; Leschot, A. Beta-glucosidases from filamentous fungi: Properties, structure, and applications. In Handbook of Carbohydrate Engineering; Yarema, K.J., Ed.; CRC Press: Boca Raton, FL, USA, 2005; pp. 645-685.

50. Jeoh, T.; Baker, J.O.; Ali, M.K.; Himmel, M.E.; Adney, W.S. Beta-D-glucosidase reaction kinetics from isothermal titration microcalorimetry. Anal. Biochem. 2005, 347, 244-253.

51. Kristensen, J.B.; Felby, C.; Jørgensen, H. Yield-determining factors in high-solids enzymatic hydrolysis of lignocellulose. Biotechnol. Biofuels 2009, 2, 11.

52. Scheller, H.V.; Ulvskov, P. Hemicelluloses. Ann. Rev. Plant Biol. 2010, 61, 263-289.

53. Gao, D.; Uppugundla, N.; Chundawat, S.P.; Yu, X.; Hermanson, S.; Gowda, K.; Brumm, P.; Mead, D.; Balan, V.; Dale, B.E. Hemicellulases and auxiliary enzymes for improved conversion of lignocellulosic biomass to monosaccharides. Biotechnol. Biofuels 2010, 4, 5.

54. Couturier, M.; Haon, M.; Coutinho, P.M., Henrissat, B.; Lesage-Meessen, L.; Berrin, J.-G. Podospora anserina hemicellulases potentiate the Trichoderma reesei secretome for saccharification of lignocellulosic biomass. Appl. Environ. Microbiol. 2011, 77, 237-246.

55. Gottschalk, L.M.F.; Oliveira, R.A.; Bon, E.P.D.S. Cellulases, xylanases, beta-glucosidase and ferulic acid esterase produced by Trichoderma and Aspergillus act synergistically in the hydrolysis of sugarcane bagasse. Biochem. Eng. J. 2010, 51, 72-78.

56. Kumar, R.; Wyman, C.E. Effect of xylanase supplementation of cellulase on digestion of corn stover solids prepared by leading pretreatment technologies. Bioresour. Technol. 2009, 100, 4203-4213.

57. Pollet, A.; Delcour, J.A.; Courtin, C.M. Structural determinants of the substrate specificities of xylanases from different glycoside hydrolase families. Crit. Rev. Biotechnol. 2010, 30, 176-191.

58. Ustinov, B.B.; Gusakov, A.V.; Antonov, A.I.; Sinitsyn, A.P. Comparison of properties and mode of action of six secreted xylanases from Chrysosporium lucknowense. Enzym. Microb. Technol. 2008, 43, 56-65.

59. Verjans, P.; Dornez, E.; Segers, M.; van Campenhout, S.; Bernaerts, K.; Beliën, T.; Delcour, J.A.; Courtin, C.M. Truncated derivatives of a multidomain thermophilic glycosyl hydrolase family 10 xylanase from Thermotoga maritima reveal structure related activity profiles and substrate hydrolysis patterns. J. Biotechnol. 2010, 145, 160-167. 
60. Jordan, D.B.; Wagschal, K. Properties and applications of microbial $\beta$-D-xylosidases featuring the catalytically efficient enzyme from Selenomonas ruminantium. Appl. Microbiol. Biotechnol. 2010, 86, 1647-1658.

61. Pastor, F.I.J.; Gallardo, O.; Sanz-Aparicio, J.; Diaz, P. Xylanases: molecular properties and applications. In Industrial Enzymes; Polaina, J., MacCabe, A.P., Eds.; Springer: Dordrecht, The Netherlands, 2007; pp. 65-82.

62. Biely, P.; Mastihubova, M.; Tenkanen, M.; Eyzaguirre, J.; Li, X.L.; Vrsanska, M. Action of xylan deacetylating enzymes on monoacetyl derivatives of 4-nitrophenyl glycosides of $\beta$-D-xylopyranose and $\alpha$-L-arabinofuranose. J. Biotechnol. 2011, 151, 137-142.

63. Koseki, T.; Fushinobu, S.; Ardiansyah, S.H.; Komai, M. Occurrence, properties, and applications of feruloyl esterases. Appl. Microbiol. Biotechnol. 2009, 84, 803-810.

64. Duranová, M.; Spániková, S.; Wösten, H.A.; Biely, P.; de Vries, R.P. Two glucuronoyl esterases of Phanerochaete chrysosporium. Arch. Microbiol. 2009, 191, 133-140.

65. Benoit, I.; Danchin, E.G.; Bleichrodt, R.J.; de Vries, R.P. Biotechnological applications and potential of fungal feruloyl esterases based on prevalence, classification and biochemical diversity. Biotechnol. Lett. 2008, 30, 387-396.

66. Saha, B.C. Alpha-L-arabinofuranosidases: biochemistry, molecular biology and application in biotechnology. Biotechnol. Adv. 2000, 18, 403-423.

67. Yip, V.L.Y.; Withers, S.G. Family 4 glycoside hydrolases are special: The first $\beta$-elimination mechanism amongst glycoside hydrolases. Biocatal. Biotransform. 2006, 24, 167-176.

68. Chong, S.L.; Battaglia, E.; Coutinho, P.M.; Henrissat, B.; Tenkanen, M.; de Vries, R.P. The alpha-glucuronidase Agu1 from Schizophyllum commune is a member of a novel glycoside hydrolase family (GH115). Appl. Microbiol. Biotechnol. 2011, 90, 1323-1332.

69. Kolenova, K.; Ryabova, O.; Vrsanska, M.; Biely, P. Inverting character of family GH115 a-glucuronidases. FEBS Lett. 2010, 584, 4063-4068.

70. Martin, K.; McDougall, B.M.; McIlroy, S.; Chen, J.Z.; Seviour, R.J. Biochemistry and molecular biology of exocellular fungal beta-(1,3)- and beta-(1,6)-glucanases. FEMS Microbiol. Rev. 2007, 31, 168-192.

71. Moreira, L.; Filho, E. An overview of mannan structure and mannan-degrading enzyme systems. Appl. Microbiol. Biotechnol. 2008, 79, 165-178.

72. Baumann, M.J. Structural evidence for the evolution of xyloglucanase activity from xyloglucan endo-transglycosylases: biological implications for cell wall metabolism. Plant Cell 2007, 19, 1947-1963.

73. Kaida, R.; Kaku, T.; Baba, K.; Oyadomari, M.; Watanabe, T.; Nishida, K.; Kanaya, T.; Shani, Z.; Shoseyov, O.; Hayashi, T. Loosening xyloglucan accelerates the enzymatic degradation of cellulose in wood. Mol. Plant 2009, 2, 904-909.

74. Lombard, V.; Bernard, T.; Rancurel, C.; Brumer, H.; Coutinho, P.M.; Henrissat, B. A hierarchical classification of polysaccharide lyases for glycogenomics. Biochem. J. 2010, 432, 437-444.

75. Payasi, A.; Sanwal, R.; Sanwal, G.G. Microbial pectate lyases: characterization and enzymological properties. World J. Microbiol. Biotechnol. 2009, 25, 1-4. 
76. Lundell, T.K.; Makela, M.R.; Hilden, K. Lignin-modifying enzymes in filamentous basidiomycetes - ecological, functional and phylogenetic review. J. Basic Microbiol. 2010, 50, 5-20.

77. Wymelenberg, A.V.; Gaskell, J.; Mozuch, M.; Sabat, G.; Ralph, J.; Skyba, O.; Mansfield, S.D.; Blanchette, R.A.; Martinez, D.; Grigoriev, I.; Kersten, P.J.; Cullen, D. Comparative transcriptome and secretome analysis of wood decay fungi Postia placenta and Phanerochaete chrysosporium. Appl. Environ. Microbiol. 2010, 76, 3599-3610.

78. Zamocky, M.; Ludwig, R.; Peterbauer, C.; Hallberg, B.M.; Divne, C.; Nicholls, P.; Haltrich, D. Cellobiose dehydrogenase - a flavocytochrome from wood-degrading, phytopathogenic and saprotropic fungi. Curr. Protein Pept. Sci. 2006, 7, 255-280.

79. Bey, M.; Berrin, J.; Poidevin, L.; Sigoillot, J.-C. Heterologous expression of Pycnoporus cinnabarinus cellobiose dehydrogenase in Pichia pastoris and involvement in saccharification processes. Microb. Cell Factories 2011, 10, 113.

80. Sweeney, M.D.; Vlasenko, E.; Abbate, E. Methods for increasing hydrolysis of cellulosic material in the presence of cellobiose dehydrogenase. U.S. Patent 20,100,159,536 A1, 24 June 2010.

81. Langston, J.A.; Shaghasi, T.; Abbate, E.; Xu, F.; Vlasenko, E.; Sweeney, M.D. Oxidoreductive cellulose depolymerization by the enzymes cellobiose dehydrogenase and glycoside hydrolase 61 . Appl. Environ. Microbiol. 2011, 77, 7007-7015.

82. Phillips, C.; Beeson, W.; Cate, J.; Marletta, M. Cellobiose dehydrogenase and a copper dependent polysaccharide monooxygenase potentiate fungal cellulose. ACS Chem. Biol. 2011, 6, 1399-1406.

83. Parawira, W.; Tekere, M. Biotechnological strategies to overcome inhibitors in lignocellulose hydrolysates for ethanol production: review. Crit. Rev. Biotechnol. 2011, 31, 20-31.

84. Bendl, R.F.; Kandel, J.M.; Amodeo, K.D.; Ryder, A.M.; Woolridge, E.M. Characterization of the oxidative inactivation of xylanase by laccase and a redox mediator. Enzym. Microb. Technol. 2008, 43, 149-156.

85. Xu, F.; Ding, H.; Tejirian, A. Detrimental effect of cellulose oxidation on cellulose hydrolysis by cellulase. Enzym. Microb. Technol. 2009, 45, 203-209.

86. Saloheimo, M.; Nakari-Setala, T.; Tenkanen, M.; Penttila, M. cDNA cloning of a Trichoderma reesei cellulase and demonstration of endoglucanase activity by expression in yeast. Eur. J. Biochem. 1997, 249, 584-591.

87. Karlsson, J.; Saloheimo, M.; Siika-Aho, M.; Tenkanen, M.; Penttila, M.; Tjerneld, F. Homologous expression and characterization of Cel61 A (EG IV) of Trichoderma reesei. Eur. J. Biochem. 2001, 268, 6498-6507.

88. Koseki, T.; Mese, Y.; Fushinobu, S.; Masaki, K.; Fujii, T.; Ito, K.; Shiono, Y.; Murayama, T.; Iefuji, H. Biochemical characterization of a glycoside hydrolase family 61 endoglucanase from Aspergillus kawachii. Appl. Microbiol. Biotechnol. 2008, 77, 1279-1285.

89. Karkehabadi, S.; Hansson, H.; Kim, S.; Piens, K.; Mitchinson, C.; Sandgren, M.L. The first structure of a glycoside hydrolase family 61 member, Cel61B from Hypocrea jecorina, at $1.6 \mathrm{~A}$ resolution. J. Mol. Biol. 2008, 383, 144-154.

90. Vaaje-Kolstad, G.; Houston, D.R.; Riemen, A.H.; Eijsink, V.G.; van Aalten, D.M. Crystal structure and binding properties of the Serratia marcescens chitin-binding protein CBP21. J. Biol. Chem. 2005, 280, 11313-11319. 
91. Harris, P.V.; Welner, D.; McFarland, K.C.; Re, E.; Navarro Poulsen, J.C.; Brown, K.; Salbo, R.; Ding, H.; Vlasenko, E.; Merino, S.; Xu, F.; Cherry, J.; Larsen, S.; Lo Leggio, L. Stimulation of lignocellulosic biomass hydrolysis by proteins of glycoside hydrolase family 61: structure and function of a large, enigmatic family. Biochemistry 2010, 49, 3305-3316.

92. Sweeney, M.D.; Vlasenko, E. Methods for determining cellulolytic enhancing activity of a polypeptide. U.S. Patent 20,100,159,494 A1, 24 June 2010.

93. Forsberg, Z.; Vaaje-Kolstad, G.; Westereng, B.; Bunæs, A.C.; Stenstrøm, Y.; MacKenzie, A.;Sørlie, M.; Horn, S.J.; Eijsink, V.G. Cleavage of cellulose by a CBM33 protein. Protein Sci. 2011, 20, 1479-1483.

94. Quinlan, R.J.; Sweeney, M.D.; Lo Leggio, L.; Otten, H.; Poulsen, J.C.; Johansen, K.S.; Krogh, K.B.; Jørgensen, C.I.; Tovborg, M.; Anthonsen, A.; et al. Insights into the oxidative degradation of cellulose by a copper metalloenzyme that exploits biomass components. Proc. Natl. Acad. Sci. USA 2011, 108, 15079-15084.

95. Westereng, B.; Ishida, T.; Vaaje-Kolstad, G.; Wu, M.; Eijsink, V.G.; Igarashi, K.; Samejima, M.; Ståhlberg, J.; Horn, S.J., Sandgren, M. The Putative Endoglucanase PcGH61D from Phanerochaete chrysosporium is a metal-dependent oxidative enzyme that cleaves cellulose. PLoS One 2011, 6, e27807.

96. Vaaje-Kolstad, G.; Westereng, B.; Horn, S.J.; Liu, Z.; Zhai, H.; Sørlie, M.; Eijsink, V.G. An oxidative enzyme boosting the enzymatic conversion of recalcitrant polysaccharides. Science 2010, 330, 219-222.

97. Sampedro, J.; Cosgrove, D.J. The expansin superfamily. Genome Biol. 2005, 6, 242.

98. Saloheimo, M.; Paloheimo, M.; Hakola, S.; Pere, J.; Swanson, B.; Nyyssönen, E.; Bhatia, A.; Ward, M.; Penttilä, M. Swollenin, a Trichoderma reesei protein with sequence similarity to the plant expansins, exhibits disruption activity on cellulosic materials. Eur. J. Biochem. 2002, 269, 4202-4211.

99. Wang, M.; Cai, J.; Huang, L.; Lv, Z.; Zhang, Y.; Xu, Z. High-level expression and efficient purification of bioactive swollenin in Aspergillus oryzae. Appl. Biochem. Biotechnol. 2010, 162, 2027-2036.

100. Jäger, G.; Girfoglio, M.; Dollo, F.; Rinaldi, R.; Bongard, H.; Commandeur, U.; Fischer, R.; Spiess, A.C.; Büchs, J. How recombinant swollenin from Kluyveromyces lactis affects cellulosic substrates and accelerates their hydrolysis. Biotechnol. Biofuels 2011, 23, 33.

101. Chen, X.A.; Ishida, N.; Todaka, N.; Nakamura, R.; Maruyama, J.; Takahashi, H.; Kitamoto, K. Promotion of efficient saccharification of crystalline cellulose by Aspergillus fumigatus Swo1. Appl. Environ. Microbiol. 2010, 76, 2556-2561.

102. Quiroz-Castañeda, R.E.; Martínez-Anaya, C.; Cuervo-Soto, L.I.; Segovia, L.; Folch-Mallol, J.L. Loosenin, a novel protein with cellulose-disrupting activity from Bjerkandera adusta. Microb. Cell Factories 2011, 10, 8.

103. Kerff, F.; Amoroso, A.; Herman, R.; Sauvage, E.; Petrella, S.; Filée, P.; Charlier, P.; Joris, B.; Tabuchi, A.; Nikolaidis, N.; Cosgrove, D.J. Crystal structure and activity of Bacillus subtilis YoaJ (EXLX1), a bacterial expansin that promotes root colonization. Proc. Natl. Acad. Sci. USA 2008, 105, 16876-16881. 
104. Lee, H.J.; Lee, S.; Ko, H.J.; Kim, K.H.; Choi, I.G. An expansin-like protein from Hahella chejuensis binds cellulose and enhances cellulase activity. Mol. Cells 2010, 29, 379-385.

105. Kim, E.S.; Lee, H.J.; Bang, W.G.; Choi, I.G.; Kim, K.H. Functional characterization of a bacterial expansin from Bacillus subtilis for enhanced enzymatic hydrolysis of cellulose. Biotechnol. Bioeng. 2009, 102, 1342-1353.

106. Georgelis, N.; Tabuchi, A.; Nikolaidis, N.; Cosgrove, D.J. Structure-function analysis of the bacterial expansin EXLX1. J. Biol. Chem. 2011, 286, 16814-16823.

107. Foreman, P.K.; Brown, D.; Dankmeyer, L.; Dean, R.; Diener, S.; Dunn-Coleman, N.S.; Goedegebuur, F.; Houfek, T.D.; England, G.J.; Kelley, A.S.; et al. Transcriptional regulation of biomass-degrading enzymes in the filamentous fungus Trichoderma reesei. J. Biol. Chem. 2003, 278, 31988-31997.

108. Foreman, P.; van Solingen, P.; Goedegebuur, F.; Ward, M. CIP1 polypeptides and their uses. U.S. Patent 7,923,235, 12 April 2011.

109. Scott, B.R.; Hill, C.; Tomashek, J.; Liu, C. Enzymatic hydrolysis of lignocellulosic feedstocks using accessory enzymes. U.S. Patent 8,017,361 B2, 13 September 2011.

110. Li, X.L.; Špániková, S.; de Vries R.P.; Biely, P. Identification of genes encoding microbial glucuronoyl esterases. FEBS Lett. 2007, 581, 4029-4035.

111. Fontes, C.M.; Gilbert, H.J. Cellulosomes: highly efficient nanomachines designed to deconstruct plant cell wall complex carbohydrates. Ann. Rev. Biochem. 2010, 79, 655-681.

112. Moraïs, S.; Barak, Y.; Hadar, Y.; Wilson, D.B.; Shoham, Y.; Lamed, R.; Bayer, E.A. Assembly of xylanases into designer cellulosomes promotes efficient hydrolysis of the xylan component of a natural recalcitrant cellulosic substrate. MBio 2011, 2, e00233-11.

113. Tsai, S.L.; Goyal, G.; Chen, W. Surface display of a functional minicellulosome by intracellular complementation using a synthetic yeast consortium and its application to cellulose hydrolysis and ethanol production. Appl. Environ. Microbiol. 2010, 76, 7514-7520.

114. Lilly, M.; Fierobe, H.P.; van Zyl, W.H.; Volschenk, H. Heterologous expression of a Clostridium minicellulosome in Saccharomyces cerevisiae. FEMS Yeast Res. 2009, 9, 1236-1249.

115. Anderson, T.D.; Robson, S.A.; Jiang, X.W.; Malmirchegini, G.R.; Fierobe, H.P.; Lazazzera, B.A.; Clubb, R.T. Assembly of minicellulosomes on the surface of Bacillus subtilis. Appl. Environ. Microbiol. 2011, 77, 4849-4858.

116. Zheng, Y.; Pan, Z.; Zhang, R. Overview of biomass pretreatment for cellulosic ethanol production. Int. J. Agric. Biol. Eng. 2009, 2, 51-68.

117. Zhang, T.; Datta, S.; Eichler, J.; Ivanova, N.; Axen, S.D.; Kerfeld, C.A.; Chen, F.; Kyrpides, N.; Hugenholtz, P.; Cheng, J.F.; Sale, K.L.; Simmons, B.; Rubin, E. Identification of a haloalkaliphilic and thermostable cellulase with improved ionic liquid tolerance. Green Chem. 2011, 13, 2083-2090.

118. Graham, J.E.; Clark, M.E.; Nadler, D.C.; Huffer, S.; Chokhawala, H.A.; Rowland, S.E.; Blanch, H.W.; Clark, D.S.; Robb, F.T. Identification and characterization of a multidomain hyperthermophilic cellulase from an archaeal enrichment. Nat. Commun. 2011, 2, 375.

(C) 2012 by the authors; licensee MDPI, Basel, Switzerland. This article is an open access article distributed under the terms and conditions of the Creative Commons Attribution license (http://creativecommons.org/licenses/by/3.0/). 